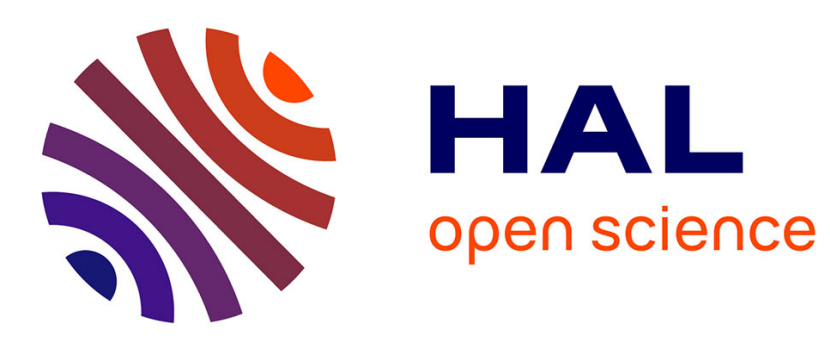

\title{
Proper Generalized Decomposition applied to linear acoustic: A new tool for broad band calculation
} Andrea Barbarulo, Pierre Ladevèze, Hervé Riou, Louis Kovalevsky

\section{To cite this version:}

Andrea Barbarulo, Pierre Ladevèze, Hervé Riou, Louis Kovalevsky. Proper Generalized Decomposition applied to linear acoustic: A new tool for broad band calculation. Journal of Sound and Vibration, 2014, 333 (11), pp.2422-2431. 10.1016/j.jsv.2014.01.014 . hal-01647860

\section{HAL Id: hal-01647860 \\ https://hal.science/hal-01647860}

Submitted on 11 May 2018

HAL is a multi-disciplinary open access archive for the deposit and dissemination of scientific research documents, whether they are published or not. The documents may come from teaching and research institutions in France or abroad, or from public or private research centers.
L'archive ouverte pluridisciplinaire HAL, est destinée au dépôt et à la diffusion de documents scientifiques de niveau recherche, publiés ou non, émanant des établissements d'enseignement et de recherche français ou étrangers, des laboratoires publics ou privés. 


\title{
Proper Generalized Decomposition applied to linear acoustic: A new tool for broad band calculation
}

\author{
Andrea Barbarulo ${ }^{a}$, Pierre Ladevèze ${ }^{\mathrm{a}, *}$, Hervé Riou ${ }^{\mathrm{a}}$, Louis Kovalevsky ${ }^{\mathrm{a}, \mathrm{b}}$ \\ a LMT-Cachan (ENS Cachan/CNRS/Paris 6 University, PRES UniverSud Paris), 61 avenue du Président Wilson, F-94230 Cachan, France \\ ${ }^{\mathrm{b}}$ University of Cambridge, Engineering Department, Trumpington Street, CB1 1PZ Cambridge, United Kingdom
}

\begin{abstract}
The calculation of the acoustic response of systems in frequency bands is becoming increasingly important in simulation-based engineering design. This is particularly true in medium-frequency bands, where the response is very sensitive to the frequency. Some standard techniques for addressing these problems present a frequency dependent formulation and may involve fixed-frequency calculations at many different frequencies. In this paper, we propose a new technique which combines the Variational Theory of Complex Rays (VTCR) with Proper Generalized Decomposition (PGD) and does not require the resolution of acoustic problems at many frequencies. In this approach, the VTCR is used to find an approximate solution of a medium-frequency acoustic problem using only a few degrees of freedom (DOFs). Then, PGD is used to find a representation of the approximate solution which is separated between two variables, the wave propagation direction and the frequency. A relevant numerical example is used to present the strategy and illustrate its applicability for frequency band calculations.
\end{abstract}

\section{Introduction}

In many industrial contexts, such as aerospace applications or car design, numerical prediction techniques are being increasingly used because they limit the need for physical prototypes to a minimum. In these industries and in the specific case of acoustic problems, engineers are often interested in the response of systems in frequency bands. The efficient calculation of the acoustic behavior of systems is the topic of this paper.

Some numerical techniques to predict the acoustic behavior of systems in frequency bands require calculations at many different fixed frequencies. This natural and straightforward approach, even with an efficient numerical tool, can easily lead to prohibitive computation times and huge needs in terms of data storage. This is particularly true in the context of medium-frequency bands because in these ranges the response is very sensitive to the frequency, which requires a very fine frequency resolution and makes the problem even worse. Therefore, for medium-frequency acoustic problems, there is a clear need to improve the efficiency of the prediction techniques for frequency bands. Some techniques have already been developed in order to do that [1-3].

In this paper, we propose an alternative approach to the development of a medium-frequency prediction techniques in frequency bands which leads to a separated representation of the unknown field. This approach uses the Variational Theory

\footnotetext{
* Corresponding author.

E-mail addresses: barbarulo@lmt.ens-cachan.fr (A. Barbarulo), ladeveze@lmt.ens-cachan.fr (P. Ladevèze), riou@lmt.ens-cachan.fr (H. Riou), 1k315@cam.ac.uk (L. Kovalevsky).
} 
of Complex Rays (VTCR) in combination with Proper Generalized Decomposition (PGD) [4,5] a promising model order reduction technique. The VTCR was introduced in [6] and belongs to the Trefftz family of methods which uses exact solutions of the governing differential equations for the expansion of the field variables.

The decisive advantage of all Trefftz methods is that since they use exact solutions of the governing equations, no refined spatial discretization inside the domain is necessary. Therefore, the model's size and the computational effort may be considerably less than with element-based methods. The VTCR differs from other Trefftz methods (such as the partition of unity method [7], the ultra-weak variational method [8], the least-squares method [9], the discontinuous enrichment method [10], the element-free Galerkin method [11], the wave boundary element method [12] or the wave-based method $[13,14])$ by the way it handles the transmission conditions at the inter-element boundaries and by the types of shape functions it uses. It has already been shown to be capable of finding accurate solutions of vibration problems involving 3D plate assemblies [15], plates with heterogeneities [16] and shell structures [17] as well as solutions of acoustic problems [18]. The VTCR is based on an original variational formulation of the problem which was developed in order to allow the approximations within elements $\Omega_{e}$ to be a priori independent of one another. Thus, in each element, any type of shape function can be used, as long as it verifies the governing Helmholtz equation. This property gives the approach great flexibility and, consequently, makes it very efficient because shape functions with a strong physical meaning related to the desired solution can be introduced without difficulty. Concerning PGD, this has been found to be an efficient technique for the resolution of multiparametric problems (problems which depend on many parameters such as the space and time problems, and the space and uncertain problems), which is what we need in order to deal with the difficulties of having to solve an acoustic problem for multiple frequencies. Therefore, even though PGD is compatible with any wave approach, a combination of PGD and the VTCR is an obvious choice when it comes to the resolution of problems in mid-frequency bands.

The VTCR has already been adapted to the handling of frequency bands in $[19,20]$. The authors proposed new algorithms for the calculation of multiple-frequency solutions, either by using a set of parameters to derive a discrete approximation of the frequency-dependent quantities within the VTCR matrix or by expanding the VTCR matrix and the right-hand side of the system to be solved into Taylor series with respect to the frequency. The objective of the third technique we are proposing here is to open a new path with regard to frequency band analysis.

The paper is structured as follows: Section 2 presents the problem being considered, which is a 2D acoustic problem driven by the Helmholtz equation. Section 3 reviews the principles of the VTCR, which leads to a discretized version of the problem. Section 4 introduces the use of PGD for frequency-dependent acoustic problems expressed in VTCR form. Section 5 illustrates the strategy in the case of a 2D acoustic problem. Conclusions and perspectives are presented in Section 6 .

\section{The reference problem}

Let us consider the general 2D interior dynamics problem of a bounded acoustic domain $\Omega$, filled with a fluid characterized by sound velocity $c_{0}$ and density $\rho_{0}$, to be studied in the frequency interval $\left.I=\right] \omega_{0}-\Delta \omega / 2 ; \omega_{0}+\Delta \omega / 2[$, where $\omega_{0}$ denotes the central frequency and $\Delta \omega$ the bandwidth of the frequency band being considered. The problem is to find $p(\mathbf{x}, \omega),(\mathbf{x}, \omega) \in \Omega \times I$ such that

$$
\mid \begin{array}{ll}
\Delta p+k^{2} p=0 & \text { over } \Omega \times I \\
p=p_{d} & \text { over } \partial_{p} \Omega \times I \\
L_{v}(p)=v_{d} & \text { over } \partial_{v} \Omega \times I \\
p-Z L_{v}(p)=h_{d} & \text { over } \partial_{Z} \Omega \times I
\end{array}
$$

where $\Delta$ is the Laplacian operator, $k=(1-i \eta) \omega / c_{0}$ is the wavenumber ( $\eta$ being the absorption coefficient), $p_{d}$ a prescribed pressure, $v_{d}$ a prescribed velocity, $Z$ a given impedance and $h_{d}$ a given function. Operator $L_{v}(\square)$ is defined by $L_{v}(\square)=\left(\mathrm{i} / \rho_{0} \omega\right)(\partial \square / \partial \mathbf{n})=\left(\mathrm{i} / \rho_{0} \omega\right) \mathbf{n}^{T} \nabla(\square)$, $\mathbf{n}$ being the outward normal and $\nabla$ the gradient operator. $\partial_{p} \Omega, \partial_{v} \Omega$ and $\partial_{Z} \Omega$ are the parts of the boundary $\partial \Omega$ of $\Omega$ where the pressure, the velocity and a Robin condition are respectively prescribed. The uniqueness of the solution of this reference problem is ensured by a strictly positive $\eta$.

If we introduce a partition of $\Omega$ into $n_{\mathrm{el}}$ non-overlapping elements $\Omega_{e}$, the following additional equations must be verified in order to ensure the continuity of the solution and its normal derivative along $\Gamma_{e, e^{\prime}}=\Omega_{e} \cap \Omega_{e^{\prime}}$ :

$$
\mid \begin{array}{ll}
p_{e}-p_{e^{\prime}}=0 & \text { along } \Gamma_{e, e^{\prime}} \times I \\
L_{v}\left(p_{e}\right)+L_{v}\left(p_{e^{\prime}}\right)=0 & \text { along } \Gamma_{e, e^{\prime}} \times I
\end{array}
$$

\section{The VTCR variational formulation of the reference problem}

The VTCR variational formulation of reference problem (1), (2) requires the definition of the functional space of the functions which satisfy the homogeneous Helmholtz governing equation, i.e. the first equation in (1):

$$
S^{e}=\left\{p_{e} ; \Delta p_{e}+k^{2} p_{e}=0 \text { over } \Omega_{e} \times I\right\}
$$


Under some nonrestrictive geometric assumptions concerning $\Omega_{e}$, for the 2D case which we are considering, these functions can be represented by waves propagating in all possible 2D directions (see $[21,22]$ ):

$$
p_{e}(\mathbf{x}, \omega) \in S^{e} \Leftrightarrow p_{e}(\mathbf{x}, \omega)=\int_{\theta=0}^{2 \pi} X_{e}(\theta, \omega) \mathrm{e}^{\mathbf{i k}_{\mathbf{e}}(\theta, \omega) \cdot \mathbf{x}} \mathrm{d} \theta
$$

where the wave vector is $\mathbf{k}_{\mathbf{e}}^{T}=k(\cos \theta, \sin \theta) . X_{e}(\theta, \omega)$, the amplitudes of the waves propagating in $\Omega_{e}$, are the unknowns of the problem.

Thus, the VTCR formulation of Problem (1), (2) is: find $\left(p_{1}, \ldots, p_{e}, \ldots, p_{n_{\mathrm{el}}}\right) \in S^{1} \times S^{e} \times \cdots \times S^{n_{\mathrm{el}}}$ such that

$$
\begin{gathered}
\sum_{\Omega_{e}} \operatorname{Re}\left\{\int_{\partial_{p} \Omega_{e}}\left(p_{e}-p_{d, e}\right) \overline{L_{V}\left[\delta p_{e}\right]} \mathrm{d} s+\int_{\partial_{v} \Omega_{e}}\left(\overline{L_{v}\left[p_{e}\right]-v_{d, e}}\right) \delta p_{e} \mathrm{~d} s\right. \\
+\frac{1}{2} \int_{\partial_{Z^{\prime}} \Omega_{e}}\left[\left(\left(1-Z_{e} L_{v}\right)\left[p_{e}\right]-h_{d, e}\right) \overline{L_{v}\left[\delta p_{e}\right]}+\left(\overline{\left(L_{v}-1 / Z_{e}\right)\left[p_{e}\right]+h_{d, e} / Z_{e}}\right) \delta p_{e}\right] \mathrm{d} s \\
\left.+\sum_{\Gamma_{e, e^{\prime}}} \frac{1}{2} \int_{\Gamma_{e^{\prime}}}\left[\left(p_{e}-p_{e^{\prime}}\right) \overline{L_{v}\left[\delta p_{e}-\delta p_{e^{\prime}}\right]}+\overline{L_{v}\left[p_{e}+p_{e^{\prime}}\right]}\left(\delta p_{e}+\delta p_{e^{\prime}}\right)\right] \mathrm{d} s\right\}=0 \\
\forall\left(\delta p_{1}, \delta p_{2}, \ldots, \delta p_{n_{\mathrm{el}}}\right) \in S^{1} \times S^{e} \times \cdots \times S^{n_{\mathrm{el}}}
\end{gathered}
$$

where $p_{d, e}, v_{d, e}$ and $h_{d, e}$ are the data we introduced in Section 2, except that now they are restricted to the boundary of $\Omega_{e}$. The overline represents the complex conjugate operation. In [23], this formulation was shown to be equivalent to the reference problem, provided there is damping.

All that is necessary in order to develop a VTCR approximation of Problem (1) and (2) is for (5) to be satisfied for a finitedimension subspace $S^{e, h}$ of $S^{e}$. Various options exist for the determination of $S^{e, h}$, i.e. for the approximation of $X_{e}(\theta, \omega)$. An overview of different choices which have been tested in 2D acoustics can be found in [23]. Here, we chose a Fourier series expansion of the wave amplitudes limited to the first $2 N_{e}+1$ terms:

$$
X_{e}(\theta, \omega)=\sum_{n=-N_{e}}^{N_{e}} X_{e}^{n}(\omega) \mathrm{e}^{\mathrm{in} \theta}
$$

where $N_{e}$ is sufficiently large to lead to a good approximate solution (the number of shape functions selected is related to their energy, see the criteria in [23]). In other words, the approximate solution consists of an expansion of $p_{e}$ over a finitedimension subspace of $\Omega_{e}$ generated by functions $\Phi_{e}^{n}(\mathbf{x}, \omega)$ :

$$
p_{e}(\mathbf{x}, \omega) \simeq \sum_{n=-N_{e}}^{N_{e}} X_{e}^{n}(\omega) \Phi_{e}^{n}(\mathbf{x}, \omega)
$$

where $\Phi_{e}^{n}(\mathbf{x}, \omega)=\int_{\theta=0}^{2 \pi} \mathrm{e}^{\mathrm{i} n \theta} \mathrm{e}^{\mathrm{i} \mathbf{k}_{\mathbf{e}}(\theta, \omega) \cdot \mathbf{x}} \mathrm{d} \theta$. At a given frequency $\omega$, the VTCR amplitude factors $X_{e}^{n}(\omega)$ are the unknown DOFs of the VTCR solution of Problem (1) and (2), and are related to the amplitudes of the waves which propagate in $\Omega_{e}$. Substituting approximate solution (7) into variational formulation (5) leads to the matrix system:

$$
\mathbf{K}(\omega) \mathbf{X}(\omega)=\mathbf{F}(\omega)
$$

where $\mathbf{K}$ and $\mathbf{F}$ denote respectively the projections of the bilinear and linear forms of (5) onto the space generated by functions $\Phi_{e}^{n}$, and $\mathbf{X}$ is the vector of the unknown contribution factors $X_{e}^{n}, e=1 \ldots n_{\mathrm{el}}, n=-N_{e} \ldots N_{e}$. Let us denote $N=\sum_{e} 2 N_{e}+1$ its size. It is important to note that matrix $\mathbf{K}$ has rather restricting numerical properties. Indeed, its coefficients are frequency-dependent, complex quantities, and it has no symmetry whatsoever. However, since the VTCR uses propagative waves instead of polynomial functions in the approximate functional spaces, the size of this matrix is very small compared to element-based methods.

\section{The use of PGD to solve VTCR problems in frequency bands}

Problem (8) is clearly defined in a multidimensional space (the 2D polar wave propagation direction and the frequency). Even if the VTCR leads to a small problem (see Section 3), this problem can lead to a great many computations in the case of a large acoustic system and a wide frequency band. This calls for the development of efficient techniques for the resolution of (8) in a frequency band. This problem was already investigated in the VTCR context in [19,20], and algorithms were proposed for the calculation of solutions for multiple frequencies either by using a set of parameters to derive a discrete approximation of the frequency-dependent quantities within the VTCR matrix or by expanding the VTCR matrix and the right-hand side of the system into Taylor series with respect to the frequency.

Here, in order to avoid having to solve large, complex systems of equations for wide frequency bands, we propose another technique based on model order reduction through a separated representation of the data. Such a representation was proposed many years ago by Ladevèze for the resolution of complex nonlinear thermomechanical problems (see [4]).

Under the name "radial approximation", it became one of the main components of the powerful nonincremental and nonlinear LArge Time INcrement (LATIN) solver [4]. More recently, a general separated representation was used in [24] to 
find approximate solutions of multidimensional partial differential equations. The separated representation was also used in [25] for the resolution of stochastic equations in which the deterministic variables and the stochastic variables were separated, very much like in [26] for the radial space-time approximation of complex multiscale problems and in [27] for finding guaranteed error bounds.

Today, the common name used for techniques involving a separated representation of the variables is Proper Generalized Decomposition (PGD). PGD belongs to the family of Reduced-Order Modeling (ROM) techniques, along with the ROM-POD method [28] and the reduced-basis element method [29], but in the case of PGD the construction of the representation takes into account the nature of the problem directly. The general form of a PGD separated representation of a function $u$ of $Q$ variables is $u\left(x_{1}, \ldots, x_{Q}\right) \simeq u^{M}\left(x_{1}, \ldots, x_{Q}\right)=\sum_{m=1}^{M} u_{m}^{1}\left(x_{1}\right) \times \cdots \times u_{m}^{N}\left(x_{Q}\right), M$ being the order of the approximation. Many applications of PGD, covering several domains, have already been presented: for example advanced nonlinear solvers using separated space-time representations; multidimensional models; the separation of physical spaces; parametric models; real-time simulations; the quantification of uncertainties and stochastic parametric analysis, etc. [30,31] give reviews of recent works on PGD. In this paper, we apply PGD to the frequency band analysis of acoustic problems.

As stated above, the innovative aspect of our approach is that it takes advantage of the PGD separated representation to solve Problem (8) in the frequency range $I=] \omega_{0}-\Delta \omega / 2 ; \omega_{0}+\Delta \omega / 2[$. Then, one tries to find an optimum approximation of $\mathbf{X}(\omega)(8)$ in the form

$$
\mathbf{X}(\omega) \simeq \mathbf{X}^{M}(\omega)=\sum_{m=1}^{M} \mathbf{X}_{m} \lambda_{m}(\omega)
$$

where $\mathbf{X}^{M}(\omega)$ (like $\mathbf{X}(\omega)$ ) is a vector defined on $\mathbb{C}^{N} \otimes \mathcal{T}(\mathcal{T}$ being the space of frequency dependent functions whose square integration on $I$ is finite), $\mathbf{X}_{m}$ are vectors of $\mathbb{C}^{N}$ and $\lambda_{m}(\omega)$ functions of $\mathcal{T}$. As explained in Section $3, \mathbf{X}^{M}(\omega)$ is related to the wave propagation in all possible 2D directions. Here, the proposed separation of the variables concerns the angular propagation direction and the frequency. Of course, none of these functions is known $a$ priori. The question is thus to find the optimal decomposition and to calculate its terms.

Many algorithms can be found in the literature to calculate quantities $\mathbf{X}_{m}$ and $\lambda_{m}(\omega)$. Some of these algorithms are reviewed and an extensive theoretical survey is presented in [25,32]. They are based on a Galerkin or a Minimal Residual formulation of the problem being considered and lead to progressive or simultaneous constructions of the decomposition. Most of these algorithms have been shown to have good convergence properties, even in the case of difficult numerical examples. Nouy [32] proposed an application of Petrov-Galerkin's algorithm to the PGD model reduction technique which improves the convergence properties of the decomposition while preserving the computational advantages of Galerkin PGD.

However, in our case, as mentioned before, matrix $\mathbf{K}(\omega)$ (see (8)) has complex coefficients and no symmetry. Therefore, among all the possible PGD algorithms, our natural choice was the Petrov-Galerkin model reduction technique, which can be viewed as a PGD method in which the orthogonality of the residuals with respect to another set of space and frequency functions is imposed, and no artificial symmetrization of the problem is required.

The Petrov-Galerkin-based PGD algorithm requires the definition of the variational formulation of the problem being considered. In our case, (8) can be expressed as: find $\mathbf{X}(\omega)$ such that

$$
\mathcal{K}(\mathbf{X}(\omega), \mathbf{Y}(\omega))=\mathcal{F}(\mathbf{Y}(\omega)) \quad \forall \mathbf{Y}(\omega) \in \mathbb{C}^{N} \otimes \mathcal{T}
$$

where $\mathcal{K}(\mathbf{X}(\omega), \mathbf{Y}(\omega))=\int_{\omega_{0}-\Delta \omega / 2}^{\omega_{0}+\Delta \omega} \mathbf{Y}(\omega)^{T} \mathbf{K}(\omega) \mathbf{X}(\omega) \mathrm{d} \omega$ and $\mathcal{F}(\mathbf{Y})=\int_{\omega_{0}-\Delta \omega / 2}^{\omega_{0}+\Delta \omega / 2} \mathbf{Y}^{T}(\omega) \mathbf{F}(\omega) \mathrm{d} \omega$. In order to develop the algorithm, let us assume that the pairs $\left\{\left(\mathbf{X}_{m}, \lambda_{m}\right)\right\}_{m=1 \ldots M-1}$ are known from the previous iteration and that we are now seeking the enrichment pair $\left(\mathbf{X}_{M}, \lambda_{M}\right)$. The method for calculating these terms consists in using the following two orthogonality criteria:

$$
\begin{array}{cc}
\mathcal{K}\left(\mathbf{X}^{M-1}+\mathbf{X}_{M} \lambda_{M}(\omega), \mathbf{Y}^{\prime} \gamma(\omega)\right)=\mathcal{F}\left(\mathbf{Y}^{\prime} \gamma(\omega)\right) & \forall \mathbf{Y}^{\prime} \in \mathbb{C}^{N} \\
\mathcal{K}\left(\mathbf{X}^{M-1}+\mathbf{X}_{M} \lambda_{M}(\omega), \mathbf{Y} \gamma^{\prime}(\omega)\right)=\mathcal{F}\left(\mathbf{Y} \gamma^{\prime}(\omega)\right) & \forall \gamma^{\prime}(\omega) \in \mathcal{T}
\end{array}
$$

$\mathbf{Y} \in \mathbb{C}^{N}$ and $\gamma(\omega) \in \mathcal{T}$ correspond to another pair of a constant vector and a frequency-dependent function respectively. Of course, additional equations must be added to (11) and (12) in order to define functions (Y, $\gamma$ ). We use the following equations:

$$
\begin{array}{cc}
\mathcal{K}\left(\mathbf{X}^{\prime} \lambda_{M}(\omega), \mathbf{Y} \gamma(\omega)\right)=\left\langle\left\langle\mathbf{X}^{\prime} \lambda_{M}(\omega), \mathbf{X}_{M} \lambda_{M}(\omega)\right\rangle\right\rangle & \forall \mathbf{X}^{\prime} \in \mathbb{C}^{N} \\
\mathcal{K}\left(\mathbf{X}_{M} \lambda^{\prime}(\omega), \mathbf{Y} \gamma(\omega)\right)=\left\langle\left\langle\mathbf{X}_{M} \lambda^{\prime}(\omega), \mathbf{X}_{M} \lambda_{M}(\omega)\right\rangle\right\rangle & \forall \lambda^{\prime}(\omega) \in \mathcal{T}
\end{array}
$$

$\langle\langle\cdot, \cdot\rangle\rangle$ denotes the inner product defined by $\langle\langle\mathbf{X} \lambda(\omega), \mathbf{Y} \gamma(\omega)\rangle\rangle=\int_{\omega_{0}-\Delta \omega / 2}^{\omega_{0}+\Delta \omega / 2} \gamma(\omega) \mathbf{Y}^{T} \mathbf{H}(\omega) \mathbf{X} \lambda(\omega) \mathrm{d} \omega$, with $\mathbf{H}(\omega)=\tilde{\mathbf{H}} \tilde{h}(\omega)$. $\tilde{\mathbf{H}}$ is a constant matrix equal to the mean value of matrix $\mathbf{K}(\omega)$ over the frequency band. $\tilde{h}(\omega)$ is a frequency-dependent function which corresponds to the mean value of the coefficients of the diagonal of $\mathbf{K}(\omega)$. With that particular choice, which is in relation with the physics of the problem, the following separation property holds:

$$
\langle\langle\mathbf{X} \lambda(\omega), \mathbf{Y} \gamma(\omega)\rangle\rangle=\left(\mathbf{X}^{T} \tilde{\mathbf{H}} \mathbf{Y}\right) \int_{\omega_{0}-\Delta \omega / 2}^{\omega_{0}+\Delta \omega / 2} \lambda(\omega) \tilde{h}(\omega) \gamma(\omega) \mathrm{d} \omega
$$

This property is useful in terms of optimal convergence (see [4,32]). Then, the idea of the algorithm is to derive the pairs $\left(\mathbf{X}_{M}, \lambda_{M}\right)$ and $(\mathbf{Y}, \gamma)$ which verify Eqs. (11)-(14) simultaneously. In order to do that, these equations are solved with a power 
iterations algorithm until convergence, i.e. until each function has reached a fixed value. If $\left(\mathbf{X}_{M}^{(q)}, \lambda_{M}^{(q)}\right)$ denotes the pair $\left(\mathbf{X}_{M}, \lambda_{M}\right)$ calculated at the current iteration with the index $(q-1)$ from the previous iteration, we use the following stopping criterion:

$$
\int_{\omega_{0}-\Delta \omega / 2}^{\omega_{0}+\Delta \omega / 2} \overline{\left(\mathbf{X}_{M}^{(q)} \lambda_{M}^{(q)}(\omega)-\mathbf{X}_{M}^{(q-1)} \lambda_{M}^{(q-1)}(\omega)\right)^{T}}\left(\mathbf{X}_{M}^{(q)} \lambda_{M}^{(q)}(\omega)-\mathbf{X}_{M}^{(q-1)} \lambda_{M}^{(q-1)}(\omega)\right) \mathrm{d} \omega<\delta_{q}
$$

where $\delta_{q}$ is related to the accuracy of this procedure.

After $\left(\mathbf{X}_{M}, \lambda_{M}\right)$ has converged, the procedure must be repeated until the convergence of the global enrichment procedure $\mathbf{X}(\omega) \simeq \mathbf{X}^{M}(\omega)=\sum_{m=1}^{M} \mathbf{X}_{m} \lambda_{m}(\omega)$. In our numerical applications, the stopping criterion was

$$
\varepsilon_{M}\left(\mathbf{X}^{M}\right)=\frac{\int_{\omega_{0}-\Delta \omega / 2}^{\omega_{0}+\Delta \omega / 2} \overline{\left(\mathbf{K}(\omega) \mathbf{X}^{M}(\omega)-\mathbf{F}(\omega)\right)^{T}}\left(\mathbf{K}(\omega) \mathbf{X}^{M}(\omega)-\mathbf{F}(\omega)\right) \mathrm{d} \omega}{\int_{\omega_{0}-\Delta \omega / 2}^{\omega_{0}-\Delta \omega / 2} \overline{\mathbf{F}(\omega)^{T}} \mathbf{F}(\omega) \mathrm{d} \omega}<\delta_{M}
$$

where $\delta_{M}$ is related to the accuracy of the PGD separated representation of the solution of Problem (8). Therefore, we introduce the following iterative algorithm:

Algorithm 1. Petrov-Galerkin approach PGD for broad band problems.

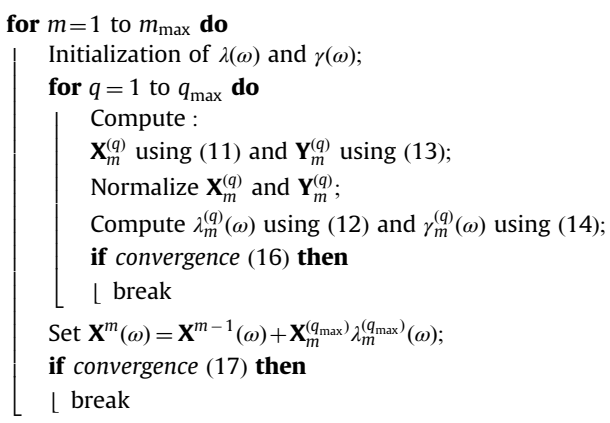

The normalization of $\mathbf{X}_{m}^{(q)}$ and $\mathbf{Y}_{m}^{(q)}$ is done with an Euclidean norm. It is important to note that several consistent initialization choices can lead to convergence, but a proper choice could make the convergence faster. This is why it is recommended to choose an initialization which is somewhat related to the problem. In the present work, we used:

$$
\lambda(\omega)=\gamma(\omega)=\sqrt{\overline{\left(\mathbf{K}(\omega) \mathbf{X}^{m-1}(\omega)-\mathbf{F}(\omega)\right)^{T}}\left(\mathbf{K}(\omega) \mathbf{X}^{m-1}(\omega)-\mathbf{F}(\omega)\right)}
$$

The most time-consuming part of the method is the resolution of (11) and (13), which are matrix problems. Eqs. (12) and (14) are scalar equations and can be solved easily and inexpensively. Thus, the proposed strategy requires the resolution of only $2 \times M \times Q$ matrix problems ( $M$ being the number of functional pairs and $Q$ the total number of internal power-type iterations required to obtain an approximate solution to the desired accuracy levels $\delta_{q}$ and $\delta_{M}$ ). In comparison, a standard incremental strategy which calculates the solution one frequency at a time requires the resolution of $N_{I}$ matrix problems ( $N_{I}$ being the number of frequencies considered for $I$ ), which, depending on the accuracy, can be extremely costly, especially in the medium-frequency range where the response is very sensitive to the data and requires a very refined frequency discretization.

\section{Numerical example}

In order to test Algorithm 1, we studied an L-shaped acoustic cavity (see Fig. 1) filled with a fluid $\left(\rho_{0}=1.25 \mathrm{~kg} / \mathrm{m}^{3}\right.$, $\left.c_{0}=340 \mathrm{~m} / \mathrm{s}, \omega \eta=0.0005\right)$ and subjected to boundary conditions in the form of a pressure $p_{d}=1 \mathrm{~Pa}$ and a velocity $v_{d}=0 \mathrm{~m} / \mathrm{s}$. Three bandwidths $\left(\Delta \omega_{1}=2 \pi \cdot 100 \mathrm{rad} / \mathrm{s}, \Delta \omega_{2}=2 \pi \cdot 200 \mathrm{rad} / \mathrm{s} \Delta \omega_{3}=2 \pi \cdot 400 \mathrm{rad} / \mathrm{s}\right)$ around the same central frequency $\omega_{0}=2 \pi \cdot 1200 \mathrm{rad} / \mathrm{s}$ were considered.

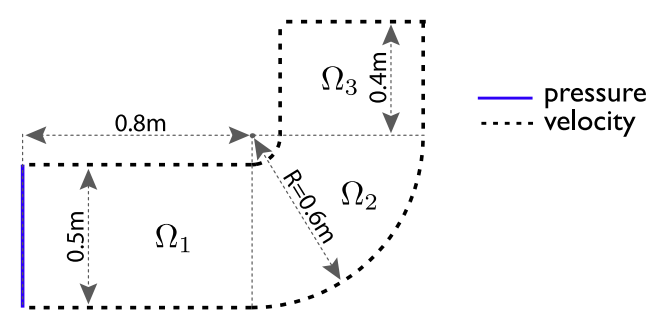

Fig. 1. Benchmark L-shaped acoustic cavity. 
As described in Section 3, this problem was solved using the VTCR. In order to do that, domain $\Omega$ was divided into 3 subdomains $\Omega_{1}, \Omega_{2}$ and $\Omega_{3}$ (see Fig. 1). An approximation of order $N_{e}=70$ (7) was used in each subdomain. It should be noted that PGD approach requires matrices $\mathbf{K}$ to have a constant size at each frequency but the order of the approximation (and therefore the size of the matrix $\mathbf{K}$ ) is frequency dependent. Thanks to the Fourier approximation nested function property, it is possible to add virtual DoFs by adding zero energy contribution functions to the approximation. In this way all matrices can be equally sized, considering the maximum number of DoFs in the band, without any degradation of its numerical properties at lower frequencies. The reference solutions for the three frequency bands considered were obtained with the VTCR using these parameters along with a very refined frequency discretization. Since our objective is to test Algorithm 1 which is based on the VTCR formulation (8), the use of a different reference, i.e. non-VTCR, would include other approximation errors, whose quantification is outside of the scope of this paper.

The iterative search for each pair of functions $\left(X_{m}, \lambda_{m}\right)$ in Algorithm 1 is controlled by two parameters: $\delta_{q}$, the stopping criterion (16), and $q_{\max }$, the maximum number of iterations. Figs. 2-4 show the error $\varepsilon_{M}\left(\mathbf{X}^{M}\right)(17)$ as a function of the number of pairs of the decomposition for different values of these parameters and for the three frequency bands $\Delta \omega_{1}$, $\Delta \omega_{2}$ and $\Delta \omega_{3}$. All the curves have similar behaviors for the different frequency bands. The weak convergence that can be noted in these figures is a standard characteristic in PGD approaches and does not affect the quality of the solution [32].

The figures on the left show that a proper criterion $\delta_{q}=0.01$ suffices to guarantee a satisfactory convergence rate. The figures on the right show that choosing a proper $q_{\max }$ also leads to convergence. This suggests that one should choose a relatively small $q_{\max }$ and stop the iterations even if the convergence criterion (16) has not been entirely satisfied for all the frequency bands. Evidence of the effectiveness of this strategy is shown in Fig. 5, which depicts a convergence comparison for the most severe case $\Delta \omega_{3}=2 \pi \cdot 400 \mathrm{rad} / \mathrm{s}$ between an algorithm stopped at a chosen value of the convergence criterion

(a)

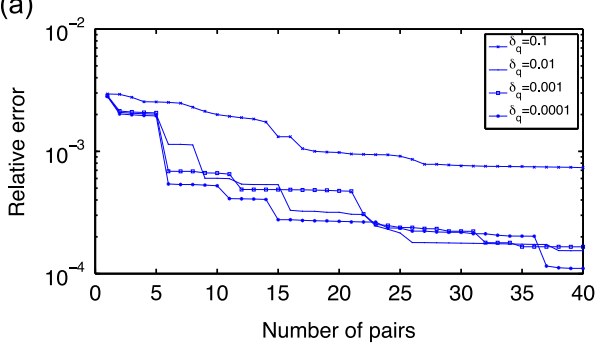

(b)

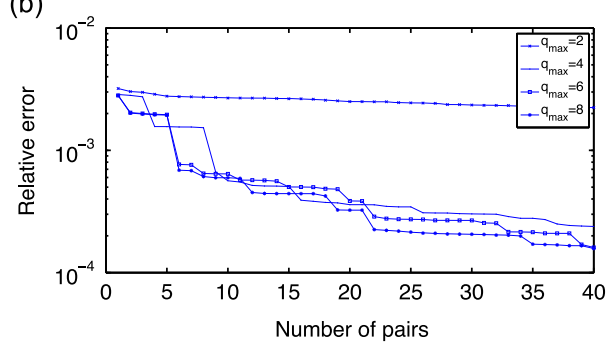

Fig. 2. The relative error $\varepsilon_{M}\left(\mathbf{X}^{M}\right)$ (17) for different $\delta_{q}$ with $q_{\max }=\infty$ (a) and for different $q_{\max }$ with $\delta_{q}=0$ (b). $\Delta \omega_{1}=2 \pi \cdot 100 \mathrm{rad} / \mathrm{s}$.

(a)

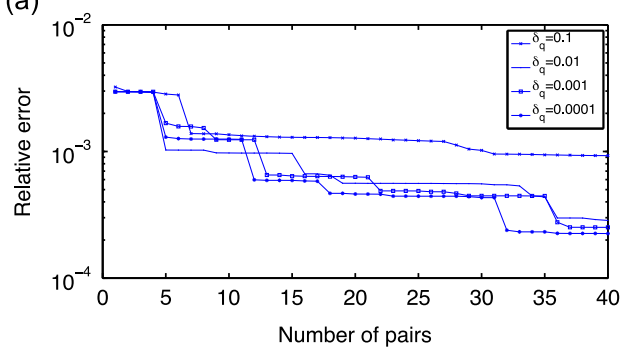

(b)

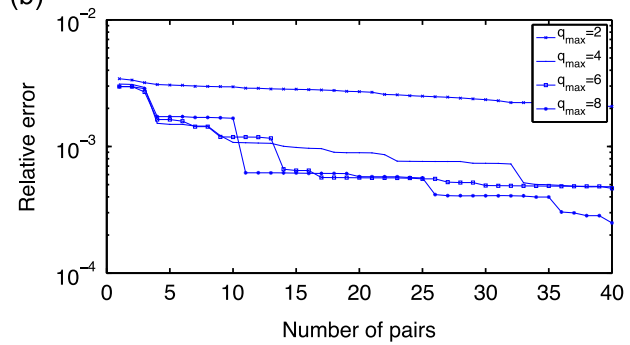

Fig. 3. The relative error $\varepsilon_{M}\left(\mathbf{X}^{M}\right)$ (17) for different $\delta_{q}$ with $q_{\max }=\infty$ (a) and for different $q_{\max }$ with $\delta_{q}=0$ (b). $\Delta \omega_{2}=2 \pi \cdot 200 \mathrm{rad} / \mathrm{s}$.

(a)

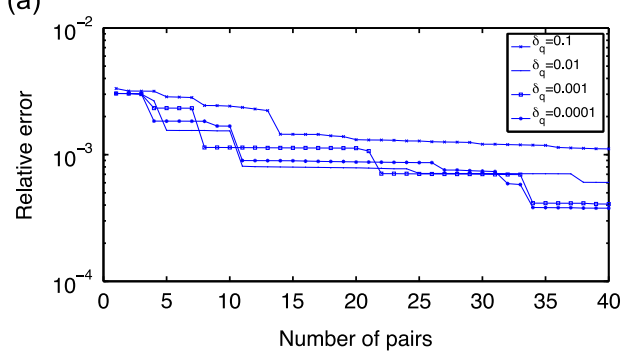

(b)

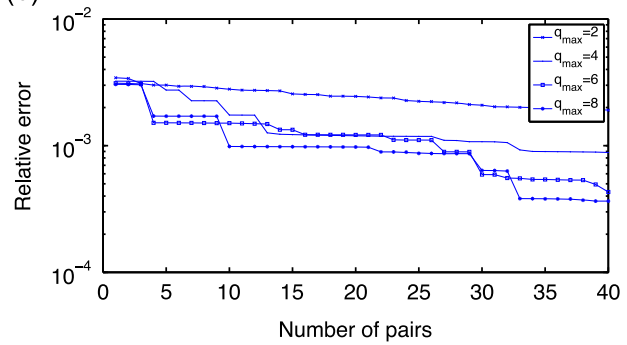

Fig. 4. The relative error $\varepsilon_{M}\left(\mathbf{X}^{M}\right)$ (17) for different $\delta_{q}$ with $q_{\max }=\infty$ (a) and for different $q_{\max }$ with $\delta_{q}=0$ (b). $\Delta \omega_{3}=2 \pi \cdot 400 \mathrm{rad} / \mathrm{s}$. 


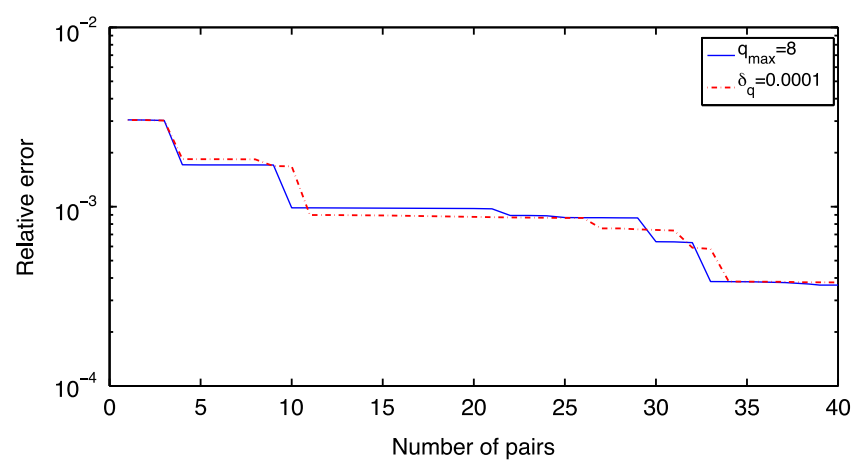

Fig. 5. The relative error $\varepsilon_{M}\left(\mathbf{X}^{M}\right)$ (17) for $\delta_{q}=0.0001$ and $q_{\max }=8 . \Delta \omega_{1}=2 \pi \cdot 400 \mathrm{rad} / \mathrm{s}$.

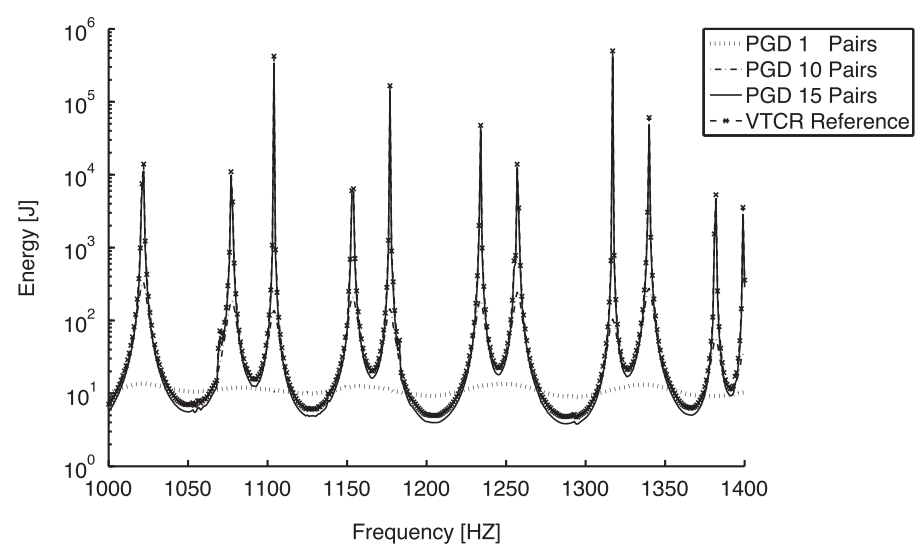

Fig. 6. Energy of the solution on the entire domain of the various approximations with different expansion orders $M$.

( $\delta_{q}=0.0001$ with $\left.q_{\max }=\infty\right)$ and the same algorithm stopped after a small specified number of iterations $\left(q_{\mathrm{max}}=8\right.$ with $\left.\delta_{q}=0\right)$. The convergence achieved with the two calculations was very similar. It took the algorithm 30 iterations to reach the required convergence criterion $\left(\delta_{q}=0.0001\right)$. This was necessary to reach full converge of the pairs, but had no decisive influence on the relative error $\varepsilon_{M}\left(\mathbf{X}^{M}\right)$ (17). Conversely, setting a small number of iterations $\left(q_{\text {max }}=8\right)$ precluded full convergence of the pairs, but still gave a satisfactory relative error $\varepsilon_{M}\left(\mathbf{X}^{M}\right)$ (17). Therefore, it is computationally more efficient not to seek full convergence, but to stop the iterations after a smaller number of iterations $q_{\max }$, thus saving on the number of iterations for each pair and, consequently, on the overall computation time.

Fig. 6 shows, for the most computationally intensive frequency band $\left(\Delta \omega_{3}=2 \pi .400 \mathrm{rad} / \mathrm{s}\right)$, a comparison of the frequency response functions obtained with our different approximations of the reference problem for various $M$ PGD pairs. The PGD solution was calculated with a coarse frequency discretization (one test point every $3 \mathrm{~Hz}$ ), and the required integrals are calculated with numerical Riemann integration. The PGD algorithm is stopped with a stoping criterion in terms of iteration $q_{\max }=8$. These frequency response functions represent the global energy of the structure. As one can see, for the frequency band considered, Approximation (9) reproduces the reference solution very well. Of course, the quality of the approximation is less good for $M$ small (although the general trend of the reference FRF is respected), but becomes better when $M$ increases. This confirms what could be anticipated from Fig. 5. With 10 PGD pairs the response is on top of the reference but the peaks, only 15 PGD pairs are needed to represent the global energy completely throughout the band.

Fig. 7 shows the approximate solution of the problem for $\omega=\omega_{0}-\Delta \omega_{3} / 2, \omega=\omega_{0}$ and $\omega=\omega_{0}+\Delta \omega_{3} / 2$ in the case of frequency band $\Delta \omega_{3}=2 \pi \cdot 400 \mathrm{rad} / \mathrm{s}$. The reference solution is also shown on the same figure. All the solutions are very similar, which illustrates the effectiveness of the proposed algorithm.

Figs. 5-7 illustrate the potential of Algorithm 1, which manages to recover the reference solution over a very large frequency band with only a few terms in Representation (9) and fewer frequency test points than the reference. From a computational point of view the trade off between the number of test points and the resolution of the full system should be considered. Frequency by frequency VTCR needs a fine frequency mesh to construct $\mathbf{K}$ matrices and then solve the full system once for frequency. On the other hand PGD-VTRC allows us to reduce the number of $\mathbf{K}$ matrices to construct but the full system has to be solved several times in order to build the desired approximation over the band. The PGD-VTCR trade off is convenient in most cases since, as shown in [33], the construction of a $\mathbf{K}$ matrix represents a major part of the total computational cost (99 percent of total solver cost) while the solution of the full system is irrelevant compared to matrix construction (less than 1 percent of total solver cost). This is particularly true since PGD solves the full system over 
(a)
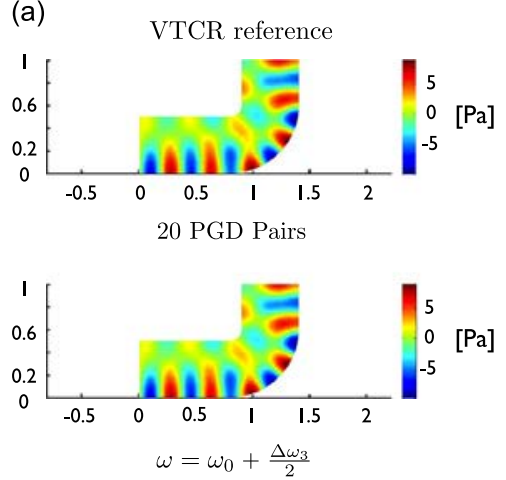

(b)
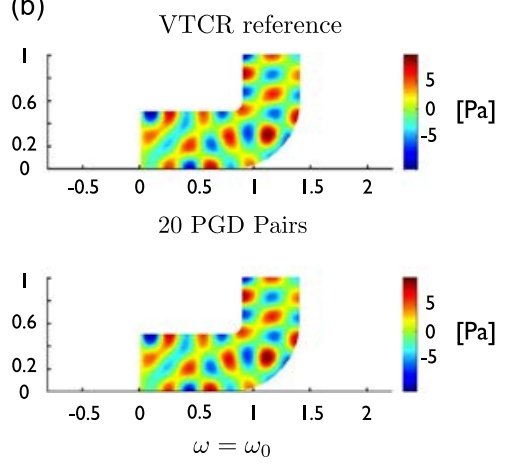

(c)

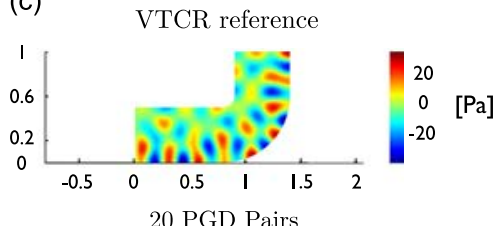

Fig. 7. Comparison of the approximate and reference real pressure fields for $\omega=\omega_{0}-\Delta \omega_{3} / 2(\mathrm{a}), \omega=\omega_{0}$ (b) and $\omega=\omega_{0}+\Delta \omega_{3} / 2$ (c).

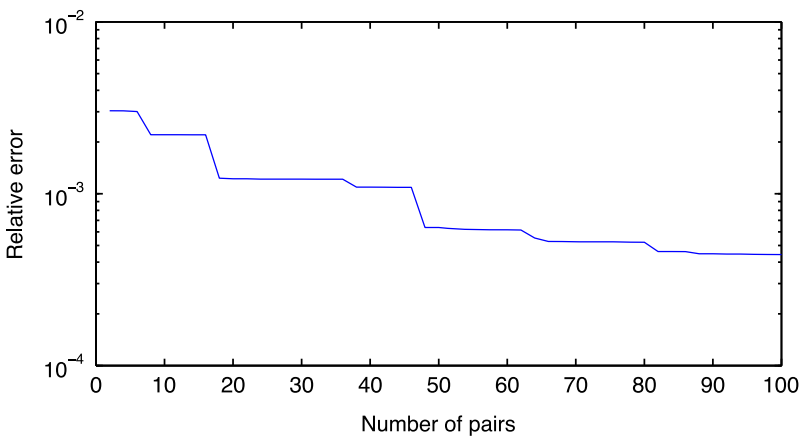

Fig. 8. The relative error $\varepsilon_{M}\left(\mathbf{X}^{M}\right)$ (17) for the second numerical example with $\Delta \omega=2 \pi \cdot 250 \mathrm{rad} / \mathrm{s}$ and $\omega_{0}=2 \pi \cdot 5275 \mathrm{rad} / \mathrm{s}$.

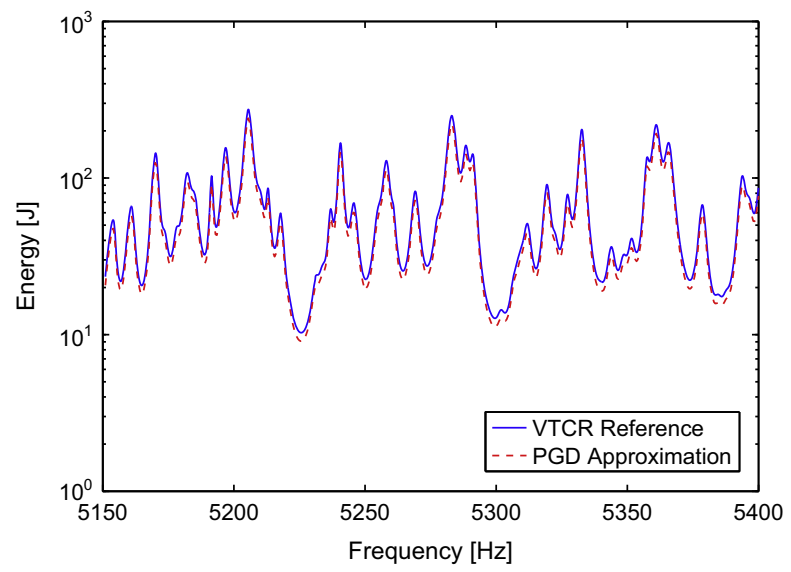

Fig. 9. Energy of the solution on the entire domain of approximations for the higher frequency band $(M=50)$, compared to VTCR reference.

a frequency band performing the integrated $\mathbf{K}$ matrix inversion only once while a standard VTCR would require one inversion for each frequency step.

In order to show the potential of the algorithm at higher frequencies a second numerical example is considered. The same geometry depicted in Fig. 1 is considered. The boundary conditions are kept the same apart from the upper side of the L shaped cavity where an impedance $Z=50-250 i \mathrm{~Pa} \mathrm{~s} / \mathrm{m}$ is prescribed. The role of this impedance is to push a midfrequency behavior inside the cavity. The considered frequency band is $\Delta \omega=2 \pi \cdot 250 \mathrm{rad} / \mathrm{s}$ around the central frequency $\omega_{0}=2 \pi \cdot 5275 \mathrm{rad} / \mathrm{s}$. Using the strategy proposed in the previous example, a $q_{\max }=8$ is chosen and one test point every $3 \mathrm{~Hz}$ is taken into account to build the PGD approximation. Convergence for this new numerical example is shown in Fig. 8. Again one can see that the relative error decreases which attests for the convergence of the method.

The convergence of the technique at higher frequencies is confirmed by the global energy comparison between a VTCR reference and an approximation with $M$ PGD pairs such that $\varepsilon_{M}\left(\mathbf{X}^{M}\right) \simeq 10^{3}$ in Fig. 9. As one can see, the strategy is able to recover the reference curve despite the complexity of the response in this frequency band. 
(a)
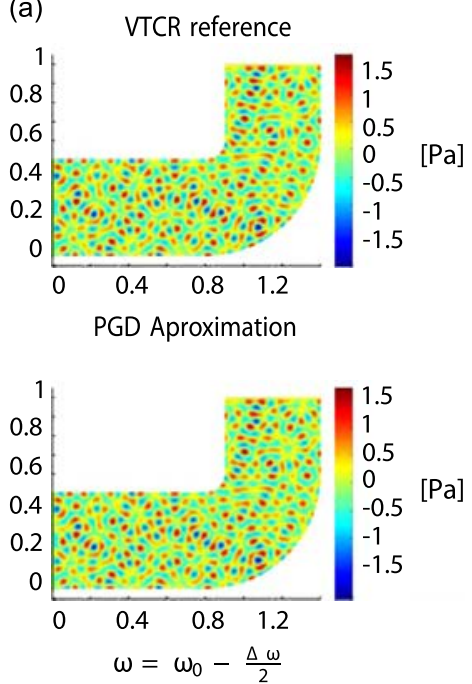

(b)
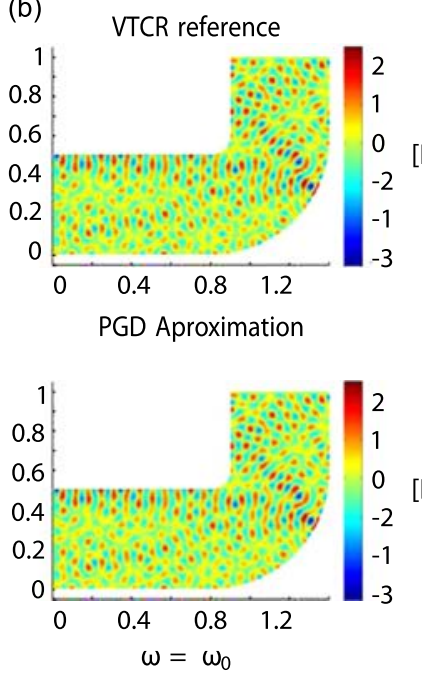

(c)

[Pa]
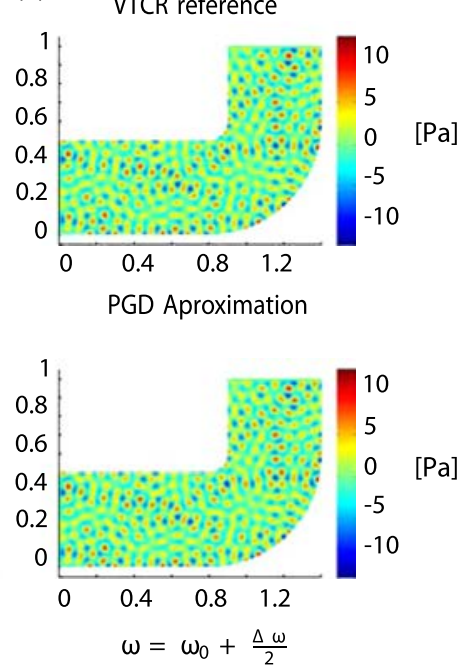

Fig. 10. Comparison of the approximate and reference real pressure fields for $\omega=\omega_{0}-\Delta \omega_{3} / 2$ (a), $\omega=\omega_{0}$ (b) and $\omega=\omega_{0}+\Delta \omega_{3} / 2$ (c).

Fig. 10 shows the approximate solution and the reference solution for this selected higher frequency band. As one can see the solutions are very similar.

\section{Conclusion}

In this paper a new version of the VTCR for the resolution of medium-frequency problems in frequency bands is proposed. It is based on its combination with the PGD. It leads to a decomposition of the solution into a basis of constant vectors and a set of frequency-dependent functions. The proposed decomposition is obtained using a power iterations algorithm. Two numerical examples showed the applicability of the method and the convergence of the algorithm. Since VTCR and PGD have been coupled for the first time in this study, the focus has been set mainly on its feasibility, rather than on its performance or limitations. In particular further analyses have to be performed to asset if PGD-VTCR advantages are kept in the presence of complex physical behavior. After these promising results, the method should be extended to more complex cases and compared with established calculation tools.

\section{Acknowledgment}

The authors gratefully acknowledge the ITN Marie Curie Project GA-214909 “MID-FREQUENCY - CAE Methodologies for Mid-Frequency Analysis in Vibration and Acoustics".

\section{References}

[1] R. Djellouli, C. Farhat, R. Tezaur, A fast method for solving acoustic scattering problems in frequency bands, Journal of Computational Physics (168) (2001) 412-432, http://dx.doi.org/10.1006/jcph.2001.6707.

[2] M. Malhotra, P.M. Pinsky, Efficient computation of multi-frequency far-field solutions of the Helmholtz equation using Pade approximation, Journal of Computational Acoustics 1 (8) (2000) 223-240, http://dx.doi.org/10.1142/S0218396X00000145.

[3] C. Soize, Medium frequency linear vibrations of anisotropic elastic structures, Recherche Aérospatiale (1983) 65-87.

[4] P. Ladevèze, Nonlinear Computational Structural Mechanics-New Approaches and Non-Incremental Methods of Calculation, Springer, Berlin, 1999.

[5] P. Ladevèze, A. Barbarulo, H. Riou, L. Kovalevsky, The variational theory of complex rays, in: MID-FREQUENCY CAE Methodologies for Mid-Frequency Analysis in Vibration and Acoustics, Leuven University Press, 2012, pp. 155-203 (Chapter 5).

[6] P. Ladevèze, A new computational approach for structure vibrations in the medium frequency range, Comptes Rendus Académie des Sciences Paris 332 (2b) (1996) 849-856.

[7] T. Strouboulis, R. Hidajat, Partition of unity method for Helmholtz equation: q-convergence for plane-wave and wave-band local bases, Applications of Mathematics 51 (2006) 181-204, http://dx.doi.org/10.1007/s10492-006-0011-0.

[8] O. Cessenat, B. Despres, Application of an ultra weak variational formulation of elliptic pdes to the two-dimensional Helmholtz problem, SIAM Journal on Numerical Analysis 35 (1998) 255-299.

[9] P. Monk, D. Wang, A least-squares method for the Helmholtz equation, Computer Methods in Applied Mechanics and Engineering 175 (1999) 121-136, http://dx.doi.org/10.1016/0045-7825(90)90121-2.

[10] C. Farhat, I. Harari, L. Franca, The discontinuous enrichment method, Computer Methods in Applied Mechanics and Engineering 190 (2001) 6455-6479, http://dx.doi.org/10.1016/S0045-7825(01)00232-8.

[11] P. Bouillard, S. Suleau, Element-free Galerkin solutions for Helmholtz problems: formulation and numerical assessment of the pollution effect, Computer Methods in Applied Mechanics and Engineering 162 (1998) 317-335, http://dx.doi.org/10.1016/S0045-7825(97)00350-2.

[12] E. Perrey-Debain, J. Trevelyan, P. Bettess, Wave boundary elements: a theoretical overview presenting applications in scattering of short waves, Engineering Analysis with Boundary Elements 28 (2004) 131-141, http://dx.doi.org/10.1016/S0955-7997(03)00127-9. 
[13] W. Desmet, B.V. Hal, P. Sas, D. Vandepitte, A computationally efficient prediction technique for the steady-state dynamic analysis of coupled vibroacoustic systems, Advances in Engineering Software 33 (2002) 527-540, http://dx.doi.org/10.1016/S0965-9978(02)00062-5.

[14] B. Van Genechten, D. Vandepitte, W. Desmet, A direct hybrid finite element-wave based modelling technique for efficient coupled vibro-acoustic analysis, Computer Methods in Applied Mechanics and Engineering 200 (5) (2011) 742-764, http://dx.doi.org/10.1016/j.cma.2010.09.017.

[15] P. Ladevèze, L. Arnaud, P. Rouch, C. Blanzé, The variational theory of complex rays for the calculation of medium-frequency vibrations, Engineering Computations (18) (2001) 193-214. http://dx.doi.org/10.1108/02644400110365879.

[16] P. Ladevèze, L. Blanc, P. Rouch, C. Blanzé, A multiscale computational method for medium-frequency vibrations of assemblies of heterogeneous plates, Computers and Structures 81 (2003) 1267-1276, http://dx.doi.org/10.1016/S0045-7949(03)00041-5.

[17] H. Riou, P. Ladevèze, P. Rouch, Extension of the variational theory of complex rays to shells for medium-frequency vibrations, Journal of Sound and Vibration 272 (1-2) (2004) 341-360, http://dx.doi.org/10.1016/S0022-460X(03)00775-2.

[18] H. Riou, P. Ladevèze, B. Sourcis, The multiscale vtcr approach applied to acoustics problems, Journal of Computational Acoustics 16 (4) (2008) 487-505, http://dx.doi.org/10.1142/S0218396X08003750.

[19] P. Ladevèze, P. Rouch, H. Riou, X. Bohineust, Analysis of medium-frequency vibrations in a frequency range, Journal of Computational Acoustics (11) (2003) 255-284. http://dx.doi.org/10.1142/S0218396X0300195X.

[20] P. Ladevèze, H. Riou, Calculation of medium-frequency vibrations over a wide frequency range, Computer Methods in Applied Mechanics and Engineering 194 (2005) 3167-3191, http://dx.doi.org/10.1016/j.cma.2004.08.009.

[21] N. Weck, Approximation by Herglotz wave functions, Mathematical Methods in the Applied Sciences 27 (2) (2004) 155-162, http://dx.doi.org/10.1002/ mma.448.

[22] R. Ochs, A version of Runge's theorem for the Helmholtz equation with applications to scattering theory, Proceedings of the Edinburgh Mathematical Society (32) (1989) 107-119. http://dx.doi.org/10.1017/S0013091500006957.

[23] L. Kovalevsky, P. Ladevèze, H. Riou, The Fourier version of the variational theory of complex rays for medium-frequency acoustics, Computer Methods in Applied Mechanics and Engineering 225-228 (2012) 142-153.

[24] A. Ammar, B. Mokdad, F. Chinesta, R. Keunings, A new family of solvers for some classes of multidimensional partial differential equations encountered in kinetic theory modeling of complex fluids, Journal of Non-Newtonian Fluid Mechanics 139 (3) (2006) 153-176, http://dx.doi.org/ 10.1016/j.jnnfm.2006.07.007.

[25] A. Nouy, A generalized spectral decomposition technique to solve a class of linear stochastic partial differential equations, Computer Methods in Applied Mechanics and Engineering 196 (45-48) (2007) 4521-4537, http://dx.doi.org/10.1016/j.cma.2007.05.016.

[26] P. Ladevèze, J.-C. Passieux, D. Néron, The LATIN multiscale computational method and the Proper Generalized Decomposition, Computer Methods in Applied Mechanics and Engineering 199 (2010) 1287-1296, http://dx.doi.org/10.1016/j.cma.2009.06.023.

[27] P. Ladevèze, L. Chamoin, On the verification of model reduction methods based on the proper generalized decomposition, Computer Methods in Applied Mechanics and Engineering 200 (2011) 2032-2047, http://dx.doi.org/10.1016/j.cma.2011.02.019.

[28] T. Lieu, C. Farhat, M. Lesoinne, Reduced-order fluid/structure modeling of a complete aircraft configuration, Computer Methods in Applied Mechanics and Engineering 195 (41-43) (2006) 5730-5742, http://dx.doi.org/10.1016/j.cma.2005.08.026.

[29] Y. Maday, E. Rønquist, A reduced-basis element method, Journal of Scientific Computing 17 (1) (2002) 447-459, http://dx.doi.org/10.1016/S1631-073X (02)02427-5.

[30] F. Chinesta, A. Ammar, E. Cueto, Recent advances and new challenges in the use of the proper generalized decomposition for solving multidimensional models, Archives of Computational Methods in Engineering 4 (17) (2010) 327-350, http://dx.doi.org/10.1007/s11831-010-9049-y.

[31] F. Chinesta, P. Ladeveze, E. Cueto, A short review on model order reduction based on proper generalized decomposition, Archives of Computational Methods in Engineering (18) (2011) 395-404. http://dx.doi.org/10.1007/s11831-011-9064-7.

[32] A. Nouy, A priori model reduction through proper generalized decomposition for solving time-dependent partial differential equations, Computer Methods in Applied Mechanics and Engineering 199 (23-24) (2010) 1603-1626, http://dx.doi.org/10.1016/j.cma.2010.01.009.

[33] L. Kovalevsky, P. Ladevèze, H. Riou, M. Bonnet, The variational theory of complex rays for three-dimensional Helmholtz problems, Journal of Computational Acoustics 20 (4) (2012). http://dx.doi.org/10.1142/S0218396X1250021X. 